\title{
NOVICE DESIGNERS' USE OF PARTITIONING STRATEGIES TO NAVIGATE THE PROTOTYPING PROCESS
}

\author{
Hansen, Camilla Arndt; \\ Deininger, Michael \\ Technical University of Denmark
}

\begin{abstract}
Prototypes can be used to create value in all phases of the product development process. Prototyping strategies that determine how prototypes are developed should therefore be considered for the prototyping process as a whole. In this paper, we analyse how partitioning strategies were used by ten novice design teams to navigate their prototyping processes during a three-week mechatronic development project. Using the 'ProtoMapping' method, their prototyping strategies were visualized and five different types of strategies identified. Four of the ten teams formally planned how to divide their product into modules that could be prototyped in parallel or planned when to integrate prototypes to test the full product. Still, the ProtoMaps showed that most of the teams used some partitioning strategies during their project even when they did not formally decide to do so. Novice designers should be supported in making such strategies for the prototyping process deliberately. Therefore, prototyping tools should be expanded to support not only individual prototyping activities but also the prototyping process.
\end{abstract}

Keywords: Prototyping, New product development, Design process, Design education

\section{Contact:}

Hansen, Camilla Arndt

Technical University of Denmark

Mechanical Engineering

Denmark

cahan@mek.dtu.dk

Cite this article: Hansen, C. A., Deininger, M. (2021) 'Novice Designers' Use of Partitioning Strategies to Navigate the Prototyping Process', in Proceedings of the International Conference on Engineering Design (ICED21), Gothenburg, Sweden, 16-20 August 2021. DOI:10.1017/pds.2021.488 


\section{INTRODUCTION}

It is widely agreed that prototyping is a crucial part of the product development process (Christie et al., 2012; Menold et al., 2017). But is prototyping a single phase in the process or an activity that can be used in many different phases? Both perspectives can be found in the existing literature. Some parts of literature refer to the 'prototyping phase' as the time period where a model is fabricated to test a design that has been specified in previous phases. For instance: "Scientists considers [sic] that the innovation processes are conventionally divided into a number of progressive steps. An important stage of a new product is the prototyping stage" (Negoescu, 2007), or "These acoustic properties are only revealed in later product development phases (e. g. prototyping phase)" (Küstner et al., 2013). As evident in the second quote, the prototyping phase is often situated late in the development process. However, other parts of literature do not confine prototyping to one time of the product development process. Rather, they argue that prototyping should be used throughout the process to test different aspects of a product; both user desirability, technical feasibility, and market viability (Menold et al., 2017). Following this view on the purpose of prototypes, the idea of a 'prototyping phase' becomes restricting and limits the potential value that prototyping can have in product development.

Prototypes have been defined as embodiments of a solution or part of a solution that helps to learn, communicate, or make decisions about a product (Lauff et al., 2018). Thus, a prototype can be both a cardboard mock-up that explores the overall dimensions of a product, a computer simulation that optimises material thickness, or a proof-of-concept prototype that does not look or work like the final product but can test whether it solves a need. All of these prototypes could be relevant for the development of the same product, but they will be relevant at different times in the development process. This research investigates how strategies for the prototyping process can be used to decide which part of a product should be tested at what points during the product development process. By analysing how ten student design teams organised their prototyping process during a mechatronic product development project, we identify five different strategies for navigating the prototyping process. We conclude that novice designers should learn both how to structure the entire prototyping process as well as individual prototyping activities. The identified navigation strategies can be used as guidance for future projects.

\subsection{Navigating the prototyping process}

In this paper, we consider prototyping as an activity that takes place throughout the development process. We believe that there is a need to make a clearer distinction between individual prototyping activities and the connected prototyping process. We define a prototyping activity as the development of a prototype for a specific purpose. The prototyping activity can be divided into four steps: Frame (defining a purpose), Build (making a prototype), Test (testing the prototype), and Act (identifying and acting on insights from the prototype) (Hansen et al., 2020). There is a need to look at prototyping as a process and an evolution rather than simply an outcome (Nelson et al., 2019). We define the prototyping process as the use of multiple prototyping activities in the development process to develop a prototyping milestone or the final product, see Figure 1.

Prototyping activity

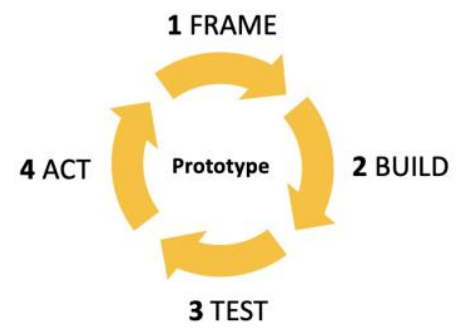

Prototyping process



Figure 1. The prototyping process consists of multiple prototyping activities

Steinert and Leifer (2012) and Menold et al. (2017) each presented models for the prototyping process. The Hunter-Gatherer Model shows how prototypes are used to explore a design direction towards 'the next big idea', but that this direction can change as the understanding of the good idea increases (Steinert and Leifer, 2012). The Hunter-Gatherer Model emphasises the unpredictable nature of new product development, but it excludes important factors that influence the development process. In larger companies, the design direction can be as much influenced by portfolio planning, launch 
timelines, validation processes, and availability of resources as by the idea itself. This dual influence between prototypes that test ideas and the development process can be seen in the Prototype for X (PfX) framework (Menold et al., 2017). This framework shows prototyping as an iterative activity that takes place in all phases of the product development process, from Planning to Production. However, it does not indicate what an actual prototyping process looks like in detail. The visualisation of concrete prototyping processes shows that these are more complex, branched, and intertwined than it appears from the PfX framework (Erichsen et al., 2020; Hansen and Özkil, 2020).

The prototyping process is defined by the goals and restrictions coming from the overarching product development process. However, the prototyping process also influences the overarching goal as each prototype leads to a deeper understanding of the feasibility, desirability and viability of a product. Navigating the prototyping process is the continuous consideration of the mutual influence between prototypes and the product development process. We introduce a new model for the prototyping process, which combines elements from the PfX framework and the Hunter-Gatherer Model to show how the prototyping process is navigated by linking the overarching project goals and individual prototyping activities, see Figure 2.

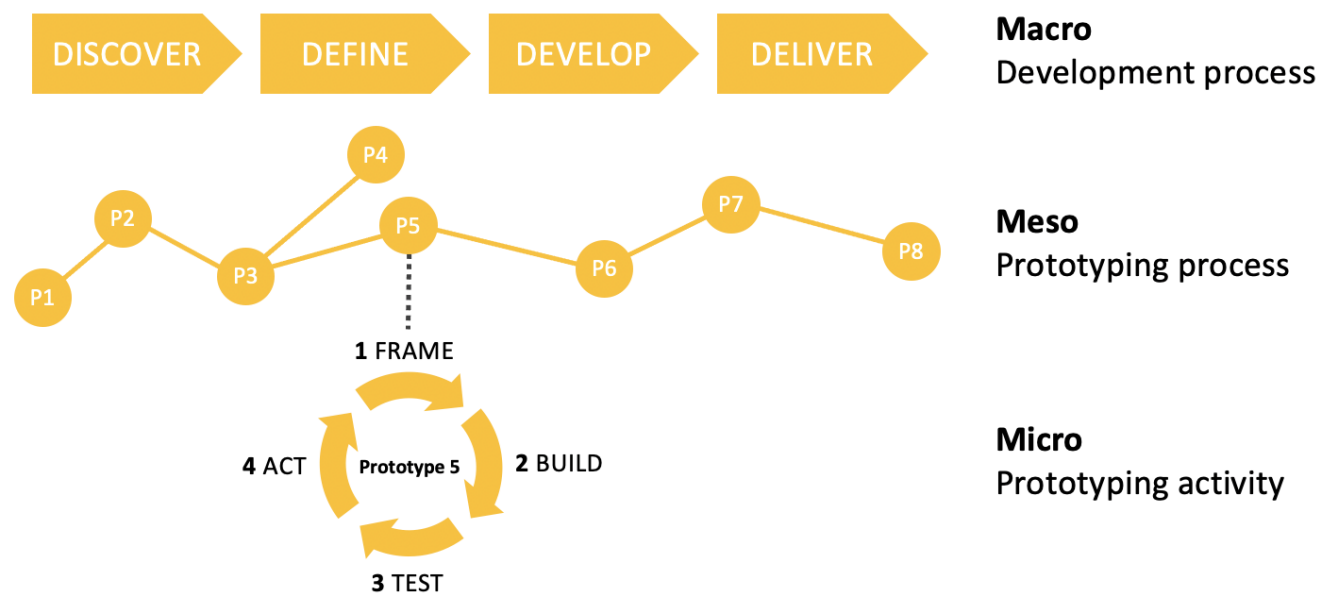

Figure 2. The three abstraction levels of the prototyping process

The new model presents prototyping at three levels of abstraction. At the Macro level, prototyping is related to the development project. Here, prototypes are often used as milestones to move towards the final product, and the detailed prototype iterations are omitted. At the Meso level, we consider which prototypes to make when, based on the goals and priorities of the project. At the Micro level, we observe the individual steps of a prototyping activity. The objective at the Micro level is to make the simplest possible prototype for a specific purpose. The Meso level connects the Micro and Macro level and shows how prototyping activities are linked together in the prototyping process to develop the final product. In this research, we analyse how students structure prototyping at a Meso level to reach their project goal.

\subsection{Strategies and support for the prototyping process}

A prototyping strategy defines how prototypes will be developed in a project (Christie et al., 2012). Examples of factors to consider in a prototyping strategy are e.g. whether to use virtual or physical prototypes, whether to scale the prototype, or whether to use similar or different materials as the final design. However, the same strategy will rarely be used for the entire project, for instance only use virtual prototypes and never any physical prototypes. When distinguishing between prototyping activity and prototyping process, one also needs to consider whether a prototyping strategy is relevant for a single prototyping activity or for a longer part of the process. There is a difference between asking: "should this prototype be virtual or physical?" and "when should we use virtual vs. physical prototypes?". The second question allows for changes in the strategy over time. Considering a partitioning strategy (Moe et al., 2004) at an activity level leads to the question: "Should we prototype a sub-system or the entire system?". At a process level we have to consider not only which part of the system to prototype when, but also whether to test sub-systems in parallel, when to integrate subsystems, and how to ensure that isolated systems can be integrated at a later stage. Thus, by only considering the prototyping strategy one prototype at a time, there is a risk that the strategies do not match the changing priorities and goals of the 
project. In this paper we focus on the use of partitioning strategies in connection to the prototyping process at the Meso level from Figure 2, i.e. the division and integration of sub-system prototypes.

Different types of design support has been developed to facilitate the use of prototyping strategies. Dunlap et al. (2014) and Camburn et al. (2013) both presented methods to help designers develop a prototyping strategy: one in the form of a heuristics-based questionnaire and the other by use of flowcharts and equations for decision-making. The Prototyping Planner (Hansen et al., 2020) and the Prototyping Canvas (Lauff et al., 2019) both present a 1-page template that helps users define a purpose and plan the minimum prototype to fulfil it. Common for all these tools and methods is that they support designers in planning and using prototyping strategies deliberately. However, they only include a single or few prototypes at a time and do not support the navigation of the holistic prototyping process.

\subsection{Research objective}

In this research, we analyse how ten student teams used the prototyping support tool Prototyping Planner during a three-week project to navigate the prototyping process and develop devices for food production. Figure 3 shows how the Prototyping Planner supports each prototyping activity at a Micro level and helps designers define a purpose for a prototype, make a build and test plan, and act on the results (Hansen et al., 2020). For every Prototyping Planner, the designer should consider the context of the prototyping activity and the product development process - however, this is not directly supported by the tool. This paper analyses the parts of the prototyping process that were not supported by the Prototyping Planner but left to the design teams themselves to organise.

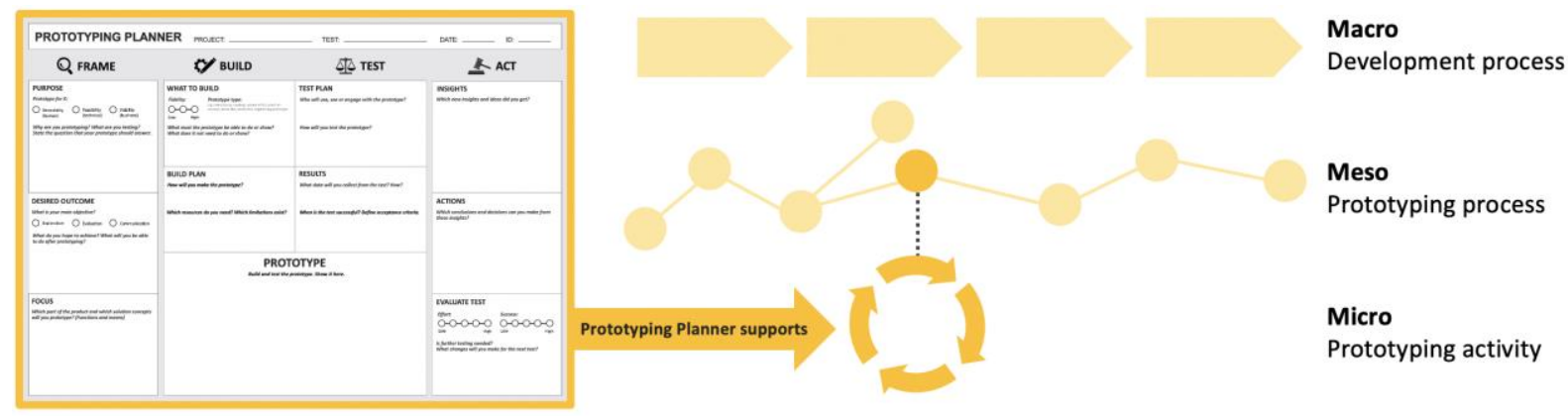

Figure 3. The Prototyping Planner (left) and the part of the prototyping process that it supports (right). Download the Prototyping Planner at www.prototypingplanner.com

We therefore asked the following research question: How did novice design teams structure their individual prototyping activities to navigate the prototyping process? We focus specifically on the strategic factor of isolating and integrating sub-systems, such as how a product was divided into different sub-systems and when prototypes were used to integrate them. By analysing how the students organised their Prototyping Planners, we aim to identify their process strategies and evaluate the need to expand the Prototyping Planner so that it not only supports the individual prototyping activities, but also the prototyping process.

\section{METHODS}

\subsection{Data collection}

Data was collected throughout a three-week mechatronic product development course at the Technical University of Denmark. Ten student teams consisting of a total of $593^{\text {rd }}$ semester engineering design students used the Prototyping Planner (V2) to plan and document their prototyping activities. The Prototyping Planner was shared between team members through Google Docs, which enabled all team members to see and work on the documents simultaneously. At the beginning of the course, the students were given an introductory lecture on prototyping and the Prototyping Planner. The rest of the course consisted primarily of developing and prototyping a device for food production, such as a tofu maker, sourdough maker, or spice dispenser. There were no requirements as to how the participants should organise their Prototyping Planners or how often they should use it. Each team also shared their project and process through a weblog. This research is based on the analysis of the ten teams' Prototyping Planners, their weblogs, and their Google Drive folders. 


\subsection{Visualising prototyping processes}

To answer the research question, we analysed how each team organised their Prototyping Planners and structured the prototyping process. This was done two times. In the first analysis, we identified formal and conscious strategies for structuring the prototyping process. For instance, one team made two Google Slides documents on their shared folder, thus indicating that they split their product into two modules (an electronic module and a mechanical module), which they prototyped in parallel. In the second analysis, we looked for structure in the prototyping process that was not formally planned by the teams. Figure 4 shows how a stack of Prototyping Planners says little about the structure of the prototyping process, while the organisation of such Prototyping Planners suddenly discloses much about the strategies for the prototyping process. This research attempts to 'connect the dots' of the Prototyping Planners.

3 week prototyping process

Idea

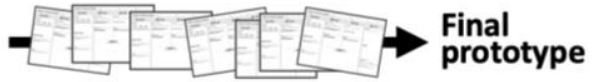

3 week prototyping process

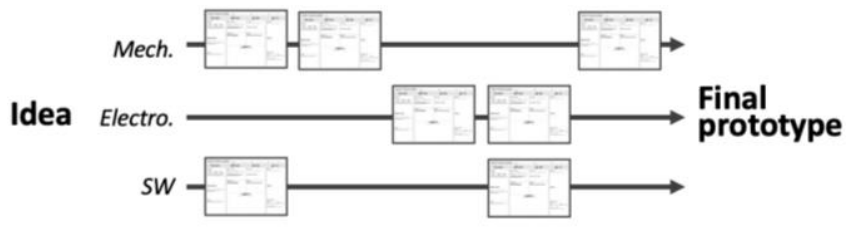

Figure 4. Left: A stack of Prototyping Planners says little about the strategies of the prototyping process. Right: Example of how the prototyping process could look if the product was divided into three modules that are prototyped in parallel.

The 'ProtoMapping' method was used to visualize the prototyping processes (Hansen and Özkil, 2020). ProtoMapping is a visualisation method which can be used to illustrate strategies used in the prototyping process based on its individual prototyping activities. In a ProtoMap, prototype iterations are placed along the horizontal axis, constituting a timeline for the process. Prototypes that test different alternative concepts or split the concept intro sub-systems are placed on the vertical axis. ProtoMapping allowed us to see which modules and parts of the product were prototyped individually and when they were integrated with each other. Figure 5 shows the ProtoMap template with some example data.

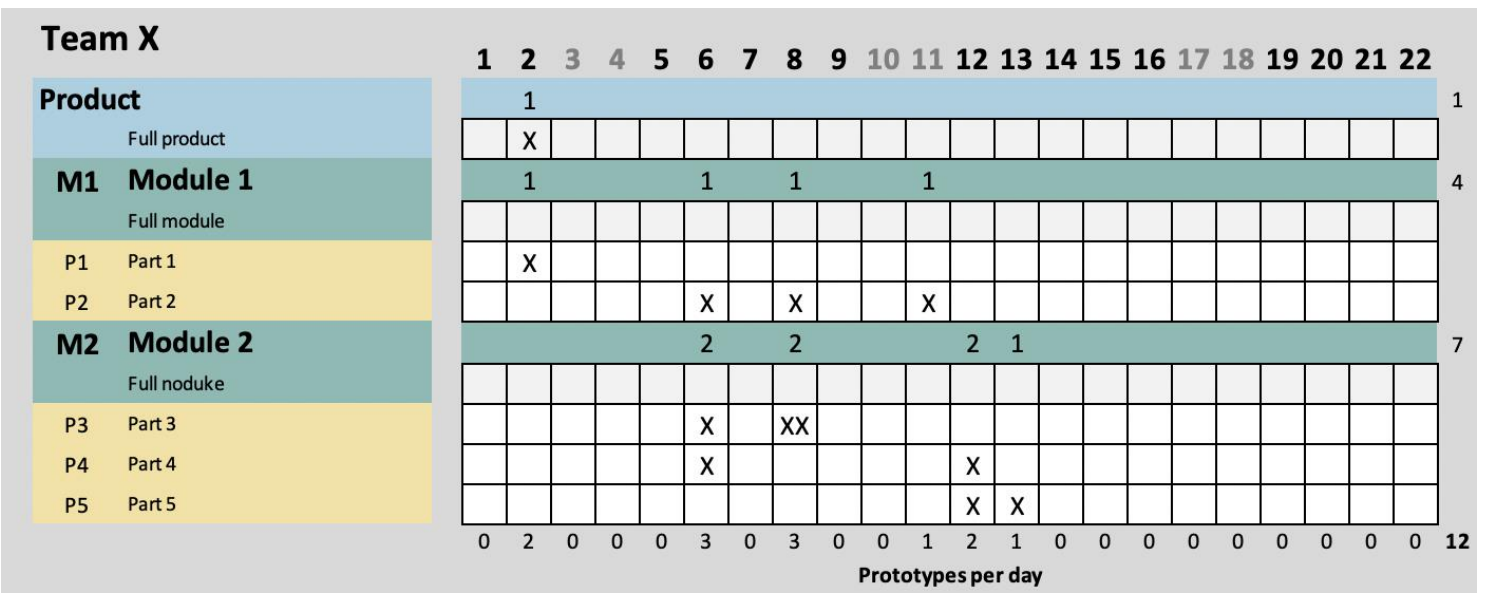

Figure 5. ProtoMap used to visualise the structure of the prototyping process. The left part shows the prototype structure: Full integrated product (blue), modules (green), and parts (yellow). ' $X$ ' marks a prototype and 'l' an integration with a prototype.

Two ProtoMaps were developed for each team; one formal ProtoMap to show the team's formal strategies and one underlying ProtoMap to show the team's underlying strategies. The formal ProtoMap was based on written documentation and the organisation of Prototyping Planners. It illustrates the team's own deliberate, conscious and planned strategies. The underlying ProtoMap was based on the content in the Prototyping Planners and shows the strategies that the teams used unplanned and spontaneously. Finally, the two ProtoMaps were compared to identify the difference between formal and underlying strategies for navigating the prototyping process. 


\section{RESULTS}

Figure 6 shows the final prototypes presented by the ten student teams at the end of the project. Oftentimes, these prototyping 'milestones' are the only part of the prototyping process that are seen by people outside the team.

Team 1

Sourdough



Team 6

Wine

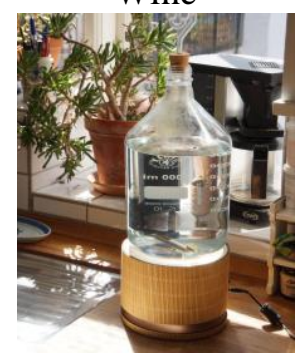

Team 2

Kefir



Team 7

Kombucha

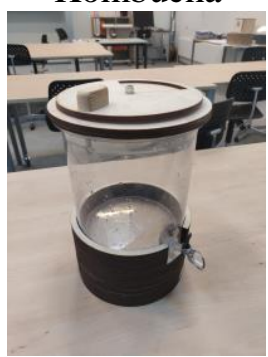

Team 3

Kimchi



Team 8

Coffee

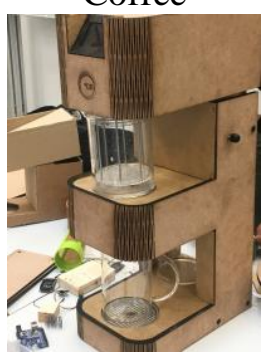

Team 4

Ultrasound mixer

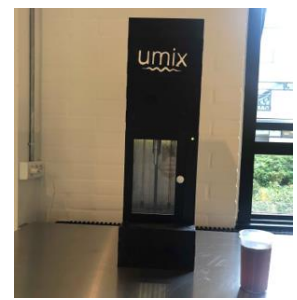

Team 9

Tofu

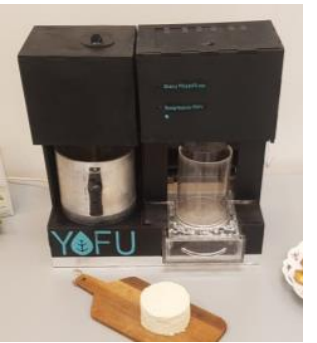

Team 5

Cheese



Team 10

Spice dispenser



Figure 6. The 10 final prototypes. This shows prototyping at a macro level of abstraction

Figure 7 shows on which days the ten student teams created prototypes using the Prototyping Planner. Here, we start to dive into the Meso level of the prototyping process. The figure shows the timing of prototypes, but still does not say much about the strategies that the teams used for their prototyping process. In the following sections, we will visualise these strategies with ProtoMaps. The first section presents the different types of partitioning strategies used by the teams while the second section compares formal and underlying strategies.



\begin{tabular}{|c|c|c|c|c|c|c|c|c|c|c|c|c|c|c|c|c|c|c|c|c|c|}
\hline 1 & 2 & 3 & 4 & 5 & 6 & 7 & 8 & 9 & 10 & 11 & 12 & 13 & 14 & 15 & 16 & 17 & 18 & 19 & 20 & 2122 & Per team \\
\hline & 1 & & & & & & & & & & 1 & 3 & 2 & & & & & & & & 7 \\
\hline & & & & & 1 & 1 & 2 & 1 & & & 1 & & & 1 & & & & & & & 7 \\
\hline 2 & 1 & & & & 2 & & & & & & 2 & & & 2 & & & & & & & 9 \\
\hline 1 & & & & 2 & & 2 & & & & & 1 & & 1 & & & 1 & & 2 & & & 10 \\
\hline 1 & & 2 & 1 & & & 2 & & & & & 1 & 1 & 2 & & & & & & & & 10 \\
\hline & & & & 3 & 1 & & 2 & 1 & & & & & 1 & & & & & & 2 & & 10 \\
\hline 1 & 2 & & & & 3 & 3 & & & & & 1 & & & & 2 & & & & & & 12 \\
\hline & & & & & & 2 & 4 & 2 & 1 & & 1 & 2 & 1 & 1 & 1 & & & 1 & & & 16 \\
\hline & 1 & & & & 3 & 2 & 3 & 1 & & 1 & 4 & 5 & & & & & & & & & 20 \\
\hline & 6 & & & & 4 & 1 & 1 & 2 & & & 2 & 7 & 4 & 3 & 1 & 1 & 1 & & 1 & & 34 \\
\hline 5 & 11 & 2 & 1 & 5 & 14 & 13 & 12 & 7 & 1 & 1 & 14 & 18 & 11 & 7 & 4 & 2 & 1 & 3 & 3 & 0 & 135 \\
\hline
\end{tabular}

Figure 7. Prototyping planners used per team per day

\subsection{Navigation strategies from ProtoMaps}

Figure 8 shows five different types of partitioning strategies used by the student teams to navigate the prototyping process. The strategies show different approaches for dividing a product into sub-parts that can be tested individually during a part of the development process. With the sub-systems strategy each prototype was used to test a different part of the product. When this was used as a formal strategy, all Prototyping Planners were found in the same slide deck or a new slide deck was created for each prototype. We consider this to be the lowest use of a formal strategy as there was no consideration of when to integrate different parts of the product with each other and only little use of iterations as merely 0-20\% of Prototyping Planner iterated a previous Prototyping Planner. The iterative sub-systems strategy is similar to the sub-systems strategy, but here product parts were iterated to a higher degree as $22-50 \%$ of Prototyping Planners referenced previous prototypes. The Prototyping Planners were also not 
organised in any particular way, but instead of seeing each prototype as a new test, the product was divided into parts that were iterated. With the modules strategy the product was not only divided into different parts, but also into different modules that were developed and prototyped in parallel. Two different ways of separating sub-systems were identified; either the modules were based on a traditional division of mechatronic products based on mechanics, electronics, and software, or they were divided based on the desired functions of the device (as known from the functions-means tree (Chakrabarti, 2002)). Team 2 divided their Kombucha maker into a mechanical and an electronic module and used a supplemental text document as a 'connector' to keep track of the tests within each module.
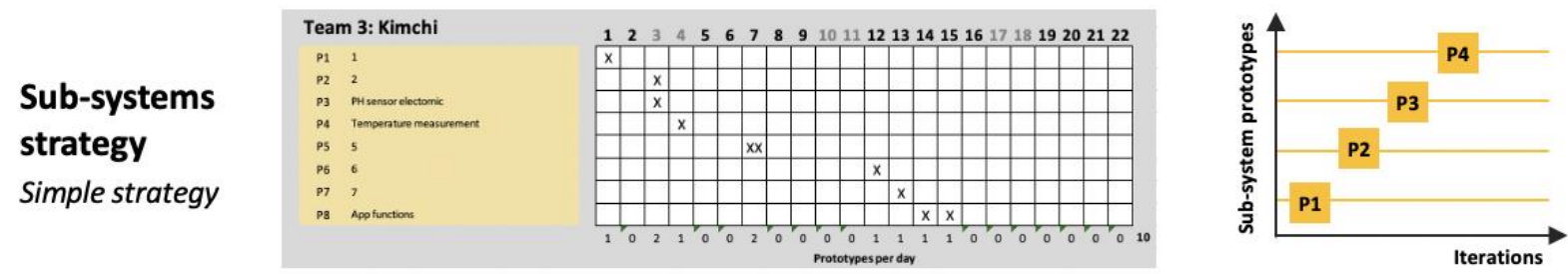

Iterative
sub-systems
strategy
Simple strategy
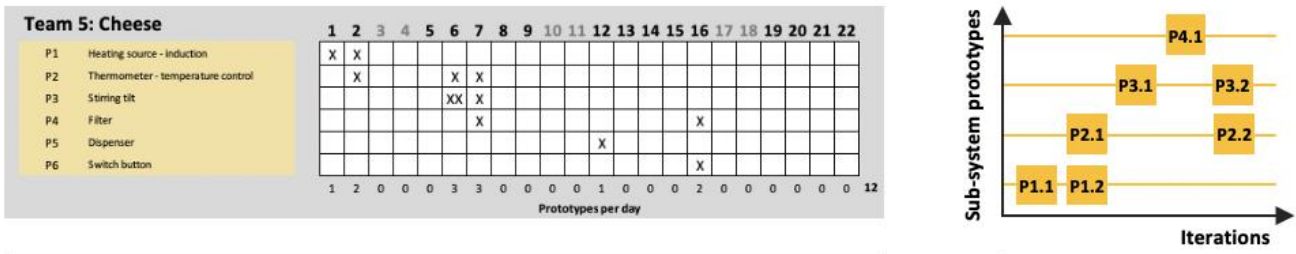

\section{Modules strategy}

Advanced strategy

\section{Integration} strategy

Advanced strategy

\section{Modules \& integration strategy}

Advanced strategy
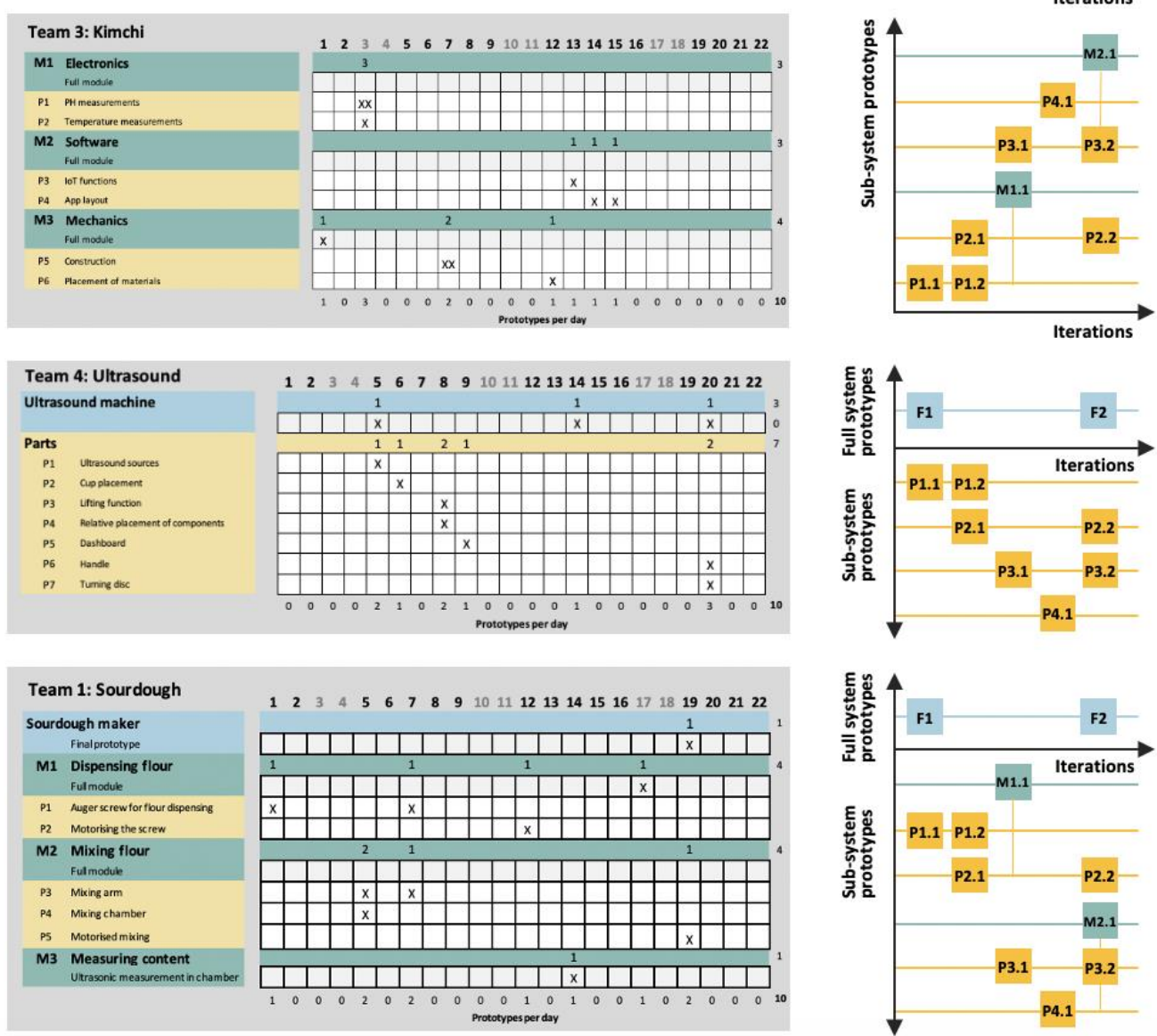

Figure 8. Examples and generic representations of the five identified partitioning strategies showing how to test the sub-systems of a product over time. All ProtoMaps can be found at www.prototypingplanner.com/protomaps

Team 4 was the only team that formally decided to use the integration strategy. Three times during the three-week course they planned a full integration prototype, which they named MVP (minimum viable product). Between these MVPs, the team prototyped sub-systems individually. Finally, the modules and integration strategy apply both sub-system modules and integration points where modules are integrated with each other. This strategy was not formally planned by any of the teams, but only found 
as an underlying strategy. Overall, we consider the sub-systems and iterative sub-systems strategies to be simple partitioning strategies while modules, integration, and modules \& integration strategies are labelled as advanced strategies.

\subsection{Comparison of formal and underlying strategies}

The same types of partitioning strategies can be identified in both the formal and underlying ProtoMaps. However, for several teams, the identified strategy changed when comparing their formal ProtoMap with their underlying ProtoMap. Details in the Prototyping Planners show how individual prototypes link together even though this is not shown in the formal documentation. When we visualised the underlying strategies, the number of modules, full system prototypes, and iterations increased, while the number of parts decreased. Figure 8 shows both types of ProtoMaps for team 3 . Formally, this team only used a sub-system strategy and did not seem to consider how they divided or integrated their prototypes. However, looking more closely at their Prototyping Planners it appears that they divided their product into electronic, software, and mechanical modules, though this strategy was not deliberately and consciously formalised. Similarly, the formal ProtoMap for team 8 shows that they did not plan when to prototype the full system. However, the underlying ProtoMap shows that almost a third of their prototyping activities were used to test their overall product, for instance to determine the relative placement of components. Table 1 presents a comparison between the strategies used in formal and underlying ProtoMaps.

Table 1. Comparison of formal and underlying strategies. The number of full prototypes, modules (Mods.), parts, and iterations (Iter.) for the teams that utilised each of the five strategies is shown. Bold font identifies where the strategy was evident

\begin{tabular}{|l|l|c|c|c|c|c|c|c|c|c|}
\hline \multirow{2}{*}{ Strategy } & \multicolumn{4}{|c|}{ Formal strategies } & \multicolumn{5}{c|}{ Underlying strategies } \\
\cline { 2 - 13 } & Teams & Full & Mod. & Parts & Iter. & Teams & Full & Mod. & Parts & Iter. \\
\hline Sub-systems & $1,3,7$ & 0 & 0 & $\mathbf{2 4}$ & 3 & - & - & - & - & - \\
\hline $\begin{array}{l}\text { Iterative sub- } \\
\text { systems }\end{array}$ & $\begin{array}{l}5,8, \\
10\end{array}$ & 0 & 0 & $\mathbf{2 3}$ & $\mathbf{1 6}$ & 5,6 & 0 & 0 & $\mathbf{8}$ & $\mathbf{1 1}$ \\
\hline Modules & $2,6,9$ & 0 & $\mathbf{4}$ & 22 & 19 & $3,9,10$ & 0 & $\mathbf{1 0}$ & 26 & 26 \\
\hline Integration & 4 & $\mathbf{3}$ & 0 & 7 & 2 & $4,7,8$ & $\mathbf{1 0}$ & 0 & 18 & 9 \\
\hline $\begin{array}{l}\text { Modules \& } \\
\text { integration }\end{array}$ & - & - & - & - & - & 1,2 & 2 & 5 & 13 & 13 \\
\hline SUM & & 3 & 8 & 82 & 53 & & $\begin{array}{c}12 \\
(+9)\end{array}$ & $\begin{array}{c}15 \\
(+7)\end{array}$ & $\begin{array}{c}65 \\
(-17)\end{array}$ & $\begin{array}{c}59 \\
(+6)\end{array}$ \\
\hline
\end{tabular}

Six of the ten teams only made little consideration of their navigation strategies, while four teams formally planned how to divide their prototype into modules or when to test fully integrated prototypes. However, the underlying ProtoMaps show that eight of the teams did use certain partitioning strategies even if they did not make formal strategic decisions. For instance, team 1 and 2 used both modules and integration prototypes even though none of the teams had explicitly planned to do so.

\section{DIscussion}

Several studies have identified the benefits of using deliberate strategies when prototyping (Christie et $a l .$, 2012). This research has analysed how teams of novice designers utilised partitioning strategies for mechatronic prototyping projects during a three-week period. Using the ProtoMapping method, we have shown both the formal and the underlying strategies used by the teams.

\subsection{Strategies for the prototyping process}

The ProtoMaps showed that five different types of partitioning strategies were used to navigate the prototyping process. Only four of the ten teams used formal strategies for their prototyping processes, while six of the teams did not formalise their overall use of prototypes during the three-week project. The four teams that formalised their prototyping strategy used a modules strategy to divide their product into modules to be prototyped in parallel or an integration strategy that determined when the full product should be tested. The other six teams did not consider how to divide their product into subsystems and when to integrate them through prototyping. However, when we look closely at the 
content of the Prototyping Planners, we find that these teams did in fact isolate, integrate and iterate more than it appeared at first sight and that some of the teams used advanced partitioning strategies even though they did not formulate them explicitly. These results show that there is a difference between novice designers' abilities to integrate and utilize design support in their projects. However, the underlying ProtoMaps shows that partitioning strategies were possible to use and relevant for all teams. Therefore, we argue that all teams would benefit from making these decisions deliberately. A team with no overall strategy for their prototyping process may make a full system prototype to integrate sub-systems, but the likelihood of them actually doing it will probably increase when they have deliberately considered this strategy. For instance, team 4 planned to make three full prototypes to test their entire product and ended up making four full system protypes. Teams 1, 2, and 7 did not have an integration strategy and only ended up making one full system prototype each.

Designers could benefit from considering how their overall product can be divided into sub-systems to test separately and when they should be integrated with other sub-systems during the prototyping process. The ProtoMaps show that the strategy for sub-system isolation and integration can change multiple times during a three-week prototyping process. Therefore, it is important to consider this strategy for the prototyping process as a whole and not just for one prototyping activity at a time. The five partitioning strategies identified in this research can be used directly to guide design teams in planning the prototyping process for the next part of their project. Borrowing from agile terminology, partitioning strategies can be used to plan the testing activities during the next 'sprint' (or cycle or phase) of the project (Schuh et al., 2018). When planning such a sprint, the design team should select one of the strategies to determine their approach for decomposing their product into sub-systems and whether integrated points should be planned. Figure 9 illustrates the use of three different strategies during three sprints in a development project.

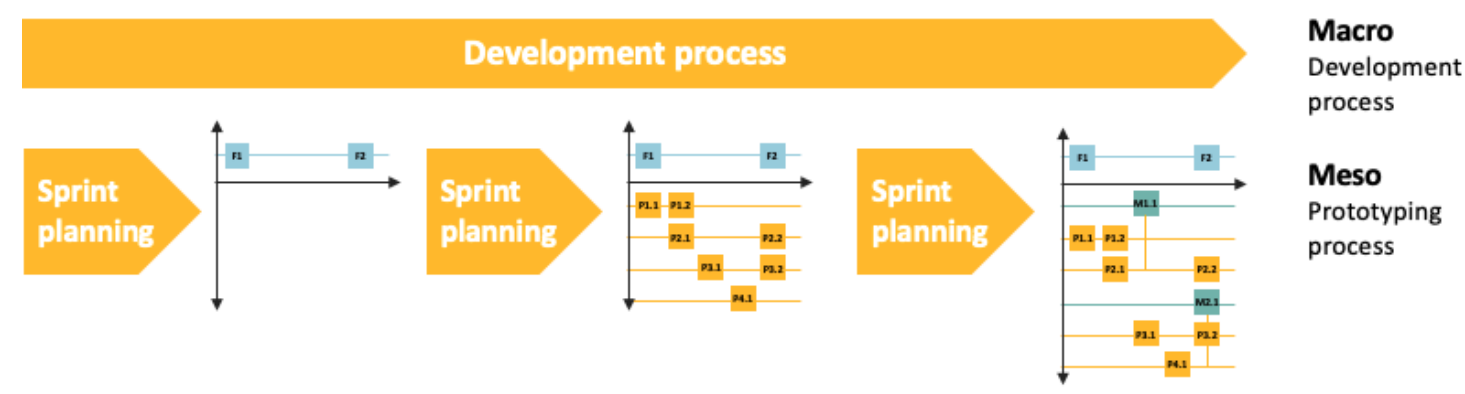

Figure 9. Partitioning strategies can be used to plan the prototyping process for a sprint

This research shows the relevance of distinguishing between prototyping activity and prototyping process. In the Introduction of this paper, we presented an expanded model for the prototyping process, showing the process at three different levels of abstraction. This paper illustrates how the Meso level of the prototyping process is needed to connect prototyping activities to the overarching development project. It also shows how the ProtoMapping method can be used to compare strategies in different prototyping processes. Based on the expanded model of the prototyping process at three levels of abstraction, it was initially considered that the Prototyping Planner only supports part of the prototyping process - namely the prototyping activities at the Micro level. This research confirms this notion and shows the need to support designers during the whole process. Thus, the Prototyping Planner should be expanded in the future to support the prototyping process as well as the individual prototyping activities.

\subsection{Limitations}

During the course, each team chose when they wished to use the Prototyping Planner. Some teams used the tool for the majority of their prototyping activities, while other teams only used it for selected activities. Therefore, the ProtoMaps do not necessarily show the prototyping processes in the full detail, though they all show enough to identify strategies at play. In general, the question of when one prototyping activity ends and the next iteration begins can be difficult to define clearly. Another important limitation is the assumption that what has not been documented by the teams was not part of their formal strategy. While we believe that formal strategic decisions would appear from the data analysis, the teams may have had discussions internally that have not been considered in the analysis. This research identified strategies for the prototyping process but did not connect the strategies to the outcome from the process. Future research should investigate the relationship between different types 
of strategies and their impact on design outcome. Finally, as this study focusses on engineering students its conclusions presently only apply to novice designers. Future studies should investigate the use of strategies in prototyping processes among expert designers in industry.

\subsection{Conclusion}

Prototyping is a process that can be observed at different levels of abstraction. This paper analysed the prototyping process at the Meso level where multiple different prototyping activities are connected to reach a prototyping milestone. Through the 'ProtoMapping' method, we demonstrated how ten teams of engineering design students used partitioning strategies to navigate the prototyping process and develop a mechatronic device for food production. Some teams used few formal strategies to go from idea to final prototype, while other teams deliberately divided their product into modules that could be prototyped separately, or planned milestones for prototyping their full concept. However, it appeared that most of the teams that did not make formal decisions about their prototyping strategies still used some of these strategies subconsciously. Novice designers should be supported in structuring the prototyping process as well as individual prototyping activities. The partitioning strategies identified in this paper can be applied by future design teams to guide the planning of their prototyping process.

\section{REFERENCES}

Camburn, B.A., Dunlap, B.U., Kuhr, R., Viswanathan, V.K., Linsey, J.S., Jensen, D.D., Crawford, R.H., et al. (2013), "Methods for Prototyping Strategies in Conceptual Phases of Design: Framework and Experimental Assessment", Proceedings of ASME IDETC/CIE 2013, presented at the IDETC/CIE, ASME, Portland, Oregon, USA, p. V005T06A033.

Chakrabarti, A. (2002), Engineering Design Synthesis: Understanding, Approaches and Tools, Springer London, London, available at: http://dx.doi.org/10.1007/978-1-4471-3717-7.

Christie, E.J., Jensen, D.D., Buckley, R.T., Menefee, D.A., Ziegler, K.K., Wood, K.L. and Crawford, R.H. (2012), "Prototyping Strategies: Literature Review and Identification of Critical Variables", ASEE Annual Conference and Exposition, Conference Proceedings, San Antonio, TX, United States, p. 25.1091.

Dunlap, B.U., Hamon, C., Camburn, B.A., Crawford, R., Jensen, D.D., Green, M., Otto, K., et al. (2014), "Heuristics-Based Prototyping Strategy Formation: Development and Tesing of a New Prototyping Planning Tool", Proceedings of the ASME 2014 IMECE, presented at the IMECE 2014.

Erichsen, J.F., Sjöman, H., Steinert, M. and Welo, T. (2020), "Protobooth: gathering and analyzing data on prototyping in early-stage engineering design projects by digitally capturing physical prototypes", Artificial Intelligence for Engineering Design, Analysis and Manufacturing, pp. 1-16.

Hansen, C.A., Jensen, L.S., Özkil, A.G. and Pacheco, N.M.M. (2020), "Fostering Prototyping Mindsets in Novice Designers with the Prototyping Planner", Proceedings of the Design Society: DESIGN Conference, Vol. 1, presented at the DESIGN 2020, pp. 1725-1734.

Hansen, C.A. and Özkil, A.G. (2020), "From Idea to Production: A Retrospective and Longitudinal Case Study of Prototypes and Prototyping Strategies", Journal of Mechanical Design, Vol. 142 No. 3, p. 031115.

Küstner, C., Breitsprecher, T. and Wartzack, S. (2013), "Design for noise reduction - The architecture of an engineering assistance system for the development of noise-reduced rotating systems", DS 75-5: Proceedings of the 19th ICED, Seoul, Korea, pp. 081-090.

Lauff, C., Menold, J. and Wood, K.L. (2019), "Prototyping Canvas: Design Tool for Planning Purposeful Prototypes", Proceedings of the Design Society: ICED, Vol. 1, pp. 1563-1572.

Lauff, C.A., Kotys-Schwartz, D. and Rentschler, M.E. (2018), "What is a Prototype? What are the Roles of Prototypes in Companies?”, Journal of Mechanical Design, Vol. 140 No. 6, p. 061102.

Menold, J., Jablokow, K. and Simpson, T. (2017), "Prototype for X (PFX): A Holistic Framework for Structuring Prototyping Methods to Support Engineering Design”, Design Studies, Vol. 50, pp. 70-112.

Moe, R.E., Jensen, D.D. and Wood, K.L. (2004), "Prototype Partitioning Based on Requirement Flexibility", presented at the ASME 2004 IDETC-CIE, ASME, pp. 65-77.

Negoescu, F. (2007), "Risk management in prototyping phase", Annals of Daaam for 2007 and Proceedings of the 18th International Daaam Symposium, DAAAM INT VIENNA, pp. 501-502.

Nelson, J., Berlin, A. and Menold, J. (2019), “ARCHIE: An Automated Data Collection Method for Physical Prototyping Efforts in Authentic Design Situations", Vol. 7, presented at the ASME 2019 IDETC/CIE, Anaheim, California, USA, available at: https://doi.org/10.1115/DETC2019-97444.

Schuh, G., Dölle, C. and Schloesser, S. (2018), "Agile Prototyping for technical systems - Towards an adaption of the Minimum Viable Product principle", Proceedings of NordDesign, presented at the NordDesign 2018, Linköping, Sweden.

Steinert, M. and Leifer, L.J. (2012), “'Finding One’s Way’: Re-Discovering a Hunter-Gatherer Model based on Wayfaring”, International Journal of Engineering Education, Vol. 28 No. 2, p. 251. 